ISSN: 0514-7336

DOI: http://dx.doi.org/10.14201/zephyrus201983187199

\title{
INSERCIÓN DE OBJETOS EN LAS PAREDES DE LA CUEVA DE LA PASIEGA B (PUENTE VIESGO, CANTABRIA)
}

\section{Insertion of objects in the walls of Pasiega B cave (Puente Viesgo, Cantabria)}

\author{
Diego Garate Maidagan*, Joseba Rios-Garaizar ${ }^{* *}$ y Sahra Talamo*** \\ * Instituto Internacional de Investigaciones Prehistóricas de Cantabria (IIIPC). Edif. Interfacultativo. Univ. de \\ Cantabria. Avda. Los Castros, s/n. 39005 Santander. Correo-e: diego.garate@unican.es. ID ORCID: 0000-0001- \\ 6685-9588 \\ ** Centro Nacional de Investigación sobre la Evolución Humana (CENIEH). Paseo Sierra de Atapuerca, 3. 09002 \\ Burgos.Correo-e: joseba.rios@cenieh.es.ID ORCID: 0000-0001-8474-2156 \\ *** Max Planck Institute for Evolutionary Anthropology. Dept. of Human Evolution. Deutscher Platz, \\ 6.04103 Leipzig (Germany). Correo-e: sahra.talamo@eva.mpg.de. ID ORCID:0000-0002-2406-3132
}

Recepción: 23/10/2018; Revisión: 22/02/2019; Aceptación: 25/03/2019

\begin{abstract}
Resumen: La inserción o depósito de objetos en las grietas, repisas y orificios de las cuevas decoradas durante el Paleolítico Superior es un fenómeno que solo ha sido objeto de investigación en los últimos años, especialmente en yacimientos situados en la vertiente norte de los Pirineos. Una prospección exhaustiva en la cueva de La Pasiega B, Puente Viesgo, Cantabria, nos ha permitido documentar evidencias similares que suman un total de 31 objetos -29 huesos y 2 sílex- en el sector más cercano a la antigua boca colmatada. El muestreo y datación de uno de los huesos nos ha permitido obtener una fecha de radiocarbono ultrafiltrada que se sitúa en el Magdaleniense Medio, en consonancia con una de las fases de decoración de la cavidad. La presencia de objetos hincados, preferentemente huesos, en áreas de hábitat de los yacimientos y compartiendo espacio con paredes decoradas es una constante que se repite en otros yacimientos como Isturitz o Gargas. No existe en la actualidad una explicación de carácter funcional para dichas evidencias, por lo que se propone una finalidad simbólica para las mismas.

Palabras clave: Magdaleniense; Cantábrico; arte; simbolismo; huesos hincados.
\end{abstract}

AвstRaCt: The interest for documenting the insertion or deposit of objects in the crevices, shelves and orifices of the decorated caves throughout the Upper Palaeolithic is quite recent and this phenomenon has almost only been documented in the Northern Pyrenean sites. During an exhaustive survey, we have identified similar evidence in the cave of La Pasiega B, Puente Viesgo, Cantabria with 31 objects -29 bones and 2 flintsin the closest sector to the old blocked entrance. The sampling and dating of one of the bones has allowed us to obtain an ultrafiltered radiocarbon date corresponding to the Middle Magdalenian, in accordance with one of the decoration phases in this sector. The presence of hinked objects, preferably bones, in areas of habitat, sharing space with decorated walls, is a constant that is repeated in other sites such as Isturitz or Gargas. There is currently no functional explanation for such evidence, so a symbolic purpose for them is proposed.

Key words: Magdalenian; Cantabrian; art; symbolism; inserted bones. 


\section{Introducción}

Durante los últimos años se ha puesto de relevancia la existencia de distintos objetos depositados y/o insertos en grietas, huecos y repisas de las paredes de las cuevas decoradas durante el Paleolítico Superior, fundamentalmente en el so de Francia (Clottes, 2007) ${ }^{1}$. En algunos casos su densidad es muy elevada, como en las cuevas pirenaicas de Gargas (Peyroux, 2011/12) y de Isturitz (Garate et al., 2013), donde superan los dos centenares. Mayoritariamente se trata de pequeños restos óseos de fauna, aunque también se han identificado elementos de industria ósea, lítica, ornamentos de hueso/diente y fragmentos de ocre. La interpretación más extendida para esta acción es la ritual, poniéndola en relación directa con la actividad artística (Bégouën y Clottes, 1995).

En la cornisa cantábrica no se ha desarrollado ninguna investigación sistemática de este tipo de evidencias, siendo la mayor parte de los hallazgos fortuitos y generalmente poco documentados (Fig. 1). En el área oriental de la región se conoce el caso de la cueva de Altxerri B, con una vértebra de bisonte hincada en una grieta a escasa distancia de otros restos de fauna en superficie datados en el Auriñaciense (Altuna, 1996; González Sainz et al., 2013). En la entrada a la gatera que lleva a la Sala de las Ciervas de la cueva de Arenaza, sobre una repisa superior, se localiza un depósito con 17 fragmentos óseos de ciervo (Gorrotxategi, 2000). Recientemente se ha detectado una diáfisis de cérvido introducida en una oquedad de la cueva de Askondo que ha sido datada directamente por ${ }^{14} \mathrm{C}$ AMs en $23760 \pm 110$ uncal вр (Beta-303671) (Garate y Rios-Garaizar, 2012). En la cueva de Astigarraga también se han localizado pequeñas esquirlas óseas e incluso una azagaya, introducidas en las grietas de las paredes (García Díez et al., 2011). A estas evidencias hay que añadir una lasca en la cueva de Lumentxa (Garate et al., 2013) y distintas evidencias localizadas en la cueva de Ekain,

1 También Peyroux, M. (2012): Les dépôts d'objets pariétaux des grottes ornées au Paléolithique supérieur: gestes, comportements symboliques, cultures. Tesis doctoral presentada en 2012 en la Univ. de Bordeaux, Francia. entre ellas una lámina de sílex localizada sobre una repisa (Altuna, 1997) o dos lápices de ocre introducidos en fisuras de la galería Auntzei².

En el resto de la cordillera, la referencia a huesos hincados o depositados en las paredes es más bien anecdótica. En la cueva de Chimeneas se señalan “... los restos parciales de un probable cérvido en una anfractuosidad del suelo [...]. Determinados huesos de las patas están aún en conexión anatómica, y es difícil admitir que los restos fueran aportados desde la entrada por la única acción del agua, ya que la galería está jalonada de numerosas concreciones..." (Groenen, 2000: 118). En la Zona IV de La Garma se ha hecho referencia a una lasca de sílex hincada en la pared (Arias, 2009). Más convencionales resultan los casos asturianos. En la cueva del Covarón, en la Sala de las Pinturas, “... se documentan: A) algunas astillas de hueso en una pequeña grieta de la pared izquierda, a 1,25 m del punto a, y 1,54 m de altura / в) fragmento de hueso largo de rumiante, en una pequeña oquedad de la pared izquierda, situada a 7,40 m del punto a, y 1,47 m de altura / c) fragmento de hueso en un testigo de arcilla adherido a la pared izquierda, a 11,90 m del punto a, y 0,75 m de altura / D) fragmento de arcilla adherido a la parte inferior de una estalagtita, en el interior del entrante situado a la derecha del hueso C...” (Arias y Pérez Suárez, 1993: 60). En Llonín también se trata de pequeños fragmentos de hueso depositados en las fisuras del "Panel Principal', además de lascas de cuarcita y sílex, e incluso un diente atrófico coloreado con ocre (Fortea et al., 2004).

La existencia de objetos introducidos en grietas de la cueva de La Pasiega в ya fue observada por uno de nosotros -D. Garate- en 2003, pero no ha sido objeto de investigación hasta 2013 y 2014. En los distintos trabajos previos sobre la cavidad solo se había mencionado una azagaya depositada en una repisa del Sector c de la cueva (Groenen, 2000).

El estudio desarrollado en la cueva de La Pasiega в ha consistido en prospectar todas las grietas, repisas y orificios de las paredes de dicho sector para detectar todas estas evidencias, documentarlas y tomar muestras para su datación por ${ }^{14} \mathrm{C}$ AMs previo

2 Observación personal de D. Garate. 


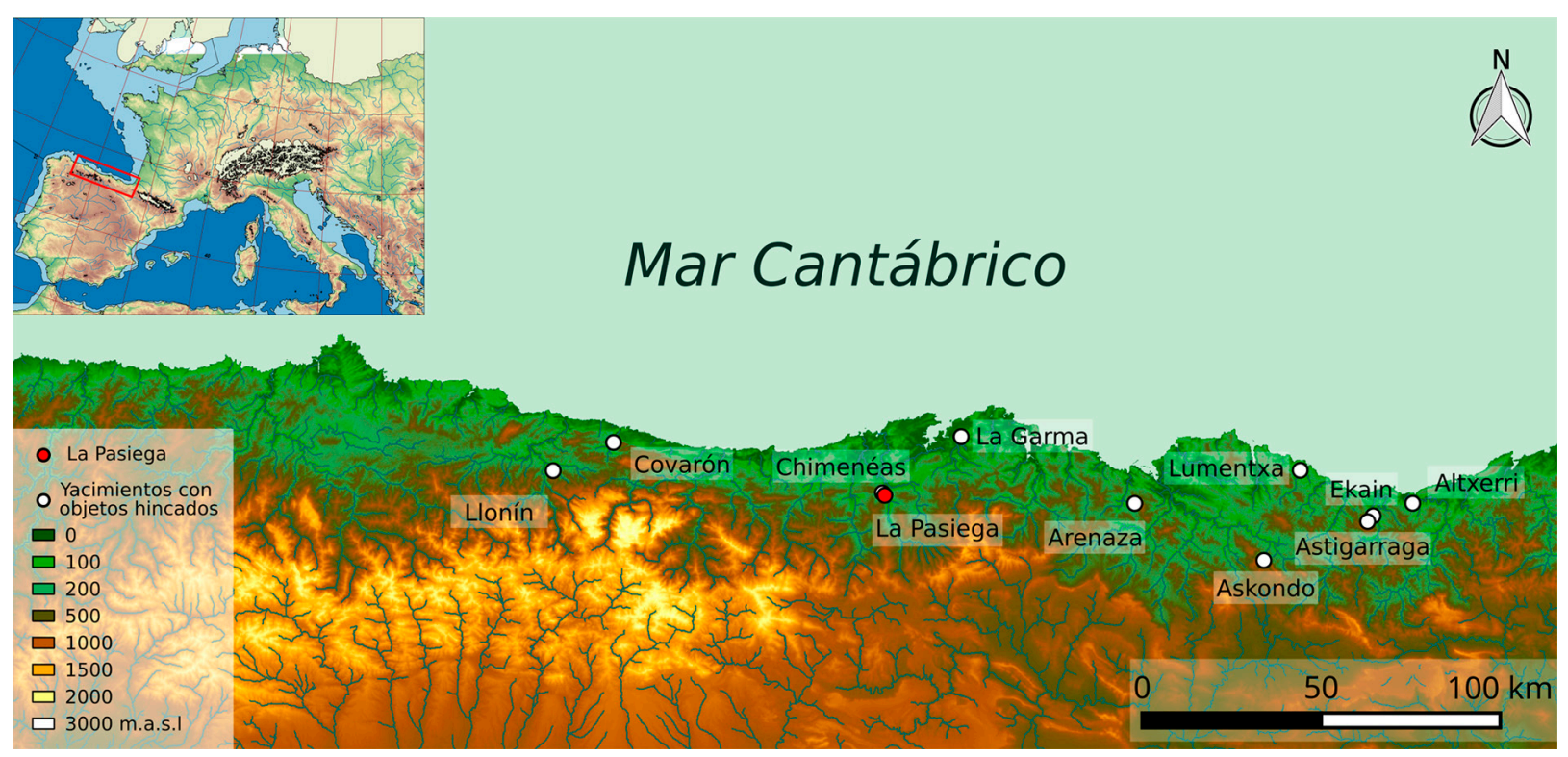

Fig. 1. Cuevas de la cornisa cantábrica con objetos insertados o depositados en las paredes (cartografía ráster obtenida de GeoEuskadi y de la European Environment Agency). Los ríos y los vectores batimétricos obtenidos de Natural Earth. La posición de los glaciares durante el LGM obtenidos de Becker et al., 2015. Mapa elaborado con QGIS 2.8 Wien e Inkscape 0.91 .

pretratamiento con ultrafiltración. Todo ello nos va a permitir contextualizar este caso particular en un marco más amplio, suponiendo una contribución importante para la comprensión global del fenómeno, tan poco conocido en la cornisa cantábrica.

\section{Materiales}

La cueva de La Pasiega se localiza en el término municipal de Puente Viesgo en Cantabria, en la margen izquierda del valle formado por el río Pas, al o de la bahía de Santander. Las bocas de acceso se encuentran a media altura en la vertiente NE del monte El Castillo, a unos $130 \mathrm{~m}$ sobre el río que, tras confluir con el Pisueña, desemboca a unos 20 km en el mar Cantábrico. Fue descubierta en 1911 por $\mathrm{H}$. Obermaier mientras se llevaban a cabo las excavaciones en la cercana cueva de El Castillo. El estudio de las manifestaciones artísticas se desarrolló y fue publicado de manera inmediata por $\mathrm{H}$. Breuil, que encabezó el análisis sistemático de las paredes; H. Obermaier, que realizó la toma de fotografías, y
H. Alcalde del Río, que se encargó de la topografía (Breuil et al., 1913).

Posteriormente, el catálogo de evidencias rupestres se ha ido incrementando con nuevos hallazgos puntuales. Como consecuencia de la apertura de dos nuevas entradas a La Pasiega, en 1951, tuvo lugar el hallazgo de varias figuras inéditas en la Galería A cerca de su confluencia con la в (González Echegaray y Ripoll, 1953/54: 60). El propio J. González Echegaray publicó en 1964 un conjunto de trece representaciones situadas en la Galería в у, por último, se dieron a conocer varios grabados y una pintura de un posible caballo localizadas en las Galerías A у в (González Echegaray y Moure, 1971). Además, se ha estudiado la relación entre la técnica y la situación topográfica de los équidos (Carayon, 1984/85: 93), la estructuración topográfica del conjunto (González García, 1987, 1990, 2001) o en concreto de la Galería a de la cueva (Heras, 1994).

En los ańos 80 del s. xx se puso en marcha un proyecto de revisión global de las cavidades, encabezado por R. Balbín Behrmann y C. González Sainz, que aportó una enorme cantidad de datos novedosos 
-triplicando el número de motivos de 284 hasta 883- (Balbín y González Sainz, 1993, 1994, 1995, 1996). Al mismo tiempo, se han aportado resúmenes actualizados de los contenidos iconográficos, de la organización topográfica y de la cronología del conjunto cárstico de La Pasiega (González Sainz y Balbín, 2000, 2003; González Sainz et al., 2003).

Recientemente, se ha procedido a la datación de costras de calcita superpuestas a algunas pinturas rojas de la cueva de La Pasiega mediante el análisis de las series de uranio (Pike et al., 2012; Hoffmann et al., 2016; Hoffmann et al., 2018).

La cueva cuenta en la actualidad con tres entradas que no se corresponden con las paleolíticas, hoy en día obturadas (Ochoa et al., 2017). Asimismo, el interior de la cavidad ha sufrido profundas modificaciones en lo que respecta a su morfología para su adaptación a las visitas turísticas, con apertura de galerías artificiales, taponado de pasos originales o instalación de escaleras, rampas o pasos de cemento. La complejidad topográfica ha sido tratada mediante la distinción de áreas diferenciables, tres en el momento de su descubrimiento y cuatro en las revisiones más recientes (Balbín y González Sainz, 1993). La Zona A comprende el tramo final de la galería principal en su extremo oriental y presenta un desarrollo de unos $25 \mathrm{~m}$. La Zona в une la anterior con una de las entradas obturadas a través de un vestíbulo que se reduce progresivamente, con varias gateras laterales y una angosta galería superior a derecha de la boca. La Zona c se restringe al sector occidental de la cueva, donde se localiza otra
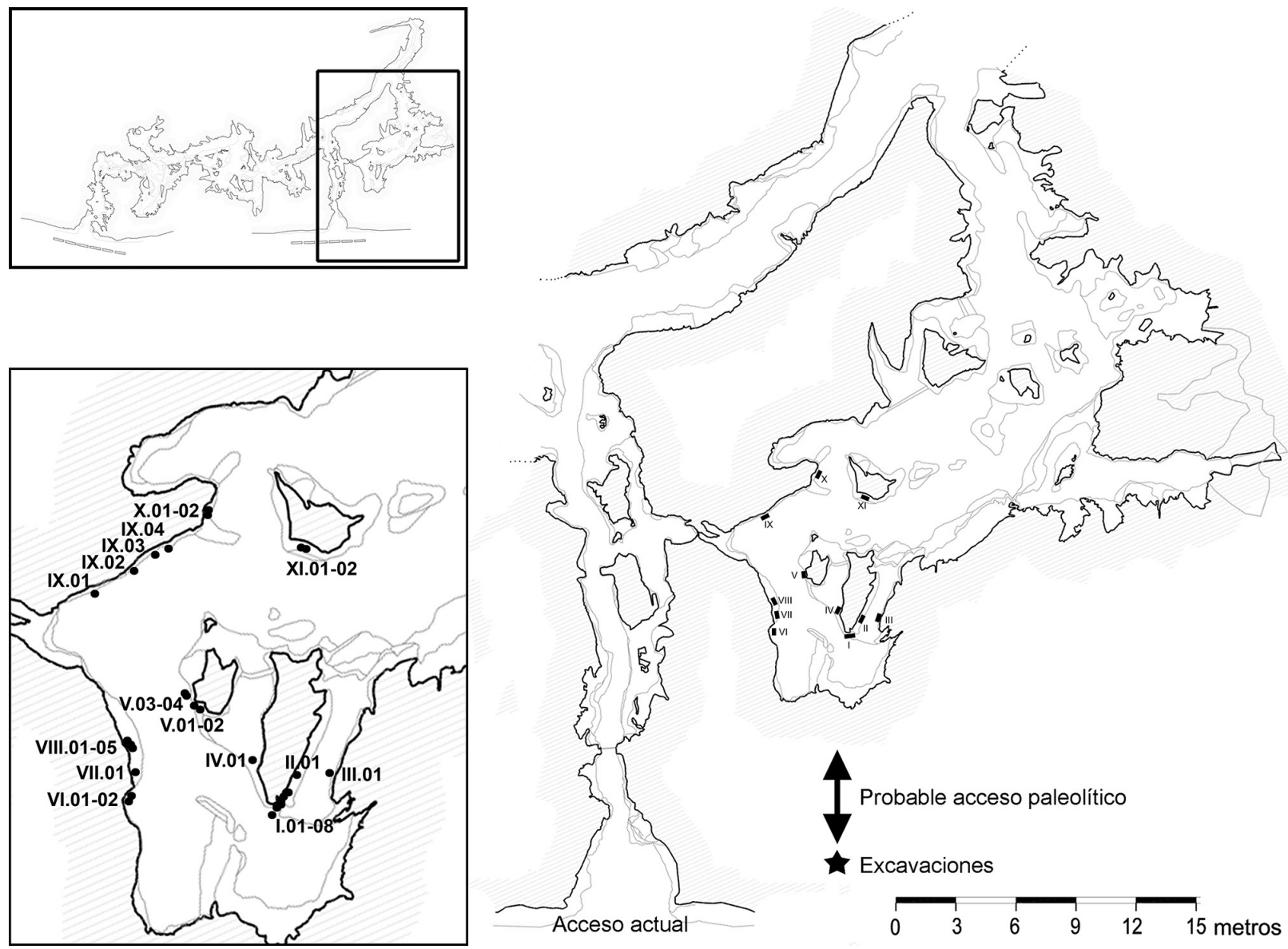

Fig. 2. Posición del Sector B en el entramado subterráneo de las cuevas de La Pasiega y de los objetos hincados localizados (a partir de Consejería de Educación, Cultura y Deporte, Gob. de Cantabria). 
de las entradas obstruidas con un vestíbulo extremadamente complejo formado por numerosos corredores naturales a diferentes alturas y dos galerías a izquierda. Por último, la Zona D abarca el pasaje de la galería principal que conecta con la A, y del que parten otras dos galerías perpendiculares, siendo una de ellas la entrada actual y la otra, con un amplio vestíbulo y una galería a un nivel superior, la entrada del descubrimiento, hoy en día obturada. Por lo tanto, en época paleolítica no era posible el tránsito por el interior de toda la cavidad. Al menos las zonas A, B y parte de la D funcionarían de manera independiente con respecto a la C y a la contigua Zona D.

Es decir, el Sector B se localiza en el extremo oriental de la cavidad, junto a una de las entradas originales hoy en día taponada por su desprendimiento (Fig. 2). A partir de esta se puede acceder a la Galería A y, desde allí, al resto de las galerías de la cavidad. En su tramo inicial, contiguo al yacimiento de ocupación, las dimensiones son muy exiguas y dan lugar a varios espacios, más o menos delimitados, con pequeños conjuntos de grafías muy perdidas. Tras el primer tramo, en el que se ubica la práctica totalidad de las grafías zoomorfas rojas, la galería da paso a una sala, no muy amplia, en la que se ha recurrido a espacios laterales exiguos y a cierta altura para realizar las decoraciones. Los huesos depositados se han localizado en distintos paneles del sector, generalmente junto a las pinturas del área más cercana a la entrada original.

La situación de las entradas originales ha dificultado el estudio de los yacimientos de ocupación. Las intervenciones realizadas están directamente relacionadas con los trabajos de adaptación de la cavidad a las visitas turísticas, dirigidas entre 1951 por J. Carballo y A. García Lorenzo (Carballo, 1952; González Echegaray y Ripoll, 1953/54). La principal intervención se desarrolló junto a la boca de entrada del Sector B, y aunque la mayor parte del material se recuperó de zonas revueltas, se llegó a describir una estratigrafía conservada en algunos puntos con tres niveles definidos. En una intervención posterior, en 1952, J. González Echegaray excavó una trinchera de 4 x 0,5 m en una zona interior; entonces, tras un manto estalagmítico, se excavaron dos niveles, uno negro y uno rojizo con escasos restos líticos (González Echegaray y Ripoll, 1953/54). Posteriormente, gracias al estudio de los materiales líticos y óseos se pudieron reconocer tres conjuntos: uno que se califica como "musteroide" (sic), con algunos restos típicos musterienses; otro que se adscribe al Solutrense, fundamentalmente a partir de la presencia de piezas foliáceas típicas. A este conjunto se atribuye también una espátula de arenisca con restos de pintura roja. Finalmente, se describe una serie magdaleniense, la más abundante del conjunto, con muchos buriles, sin laminillas de dorso y con algunos elementos característicos de industria ósea. A este nivel se atribuye un fragmento de maxilar humano. Entre los restos de fauna de todos los niveles hay ciervo, gran bóvido, caballo y oso de las cavernas (González Echegaray y Ripoll, 1953/54).

\section{Métodos}

La prospección ha consistido en el reconocimiento sistemático y detenido de las superficies rocosas organizadas por tramos para articular su desarrollo, desde la colmatación del acceso original del Sector в hasta su conexión con el Sector A, excepto las salas superiores denominadas в.7 y B.8. Para ello se ha recurrido a iluminación fría de distintas intensidades y en ningún caso se ha procedido al contacto físico directo con las paredes y techos de la cavidad, excepto durante la toma de muestras. La labor de toma de datos se ha desarrollado in situ de una manera concisa mediante el registro macrofotográfico y la observación visual con ayuda de una lupa portátil Dino-lite ${ }^{\circledR}$ AD-7013MZT 30x-200x. Los datos se registraron mediante fichas normalizadas, combinando tanto los propios del objeto como los de su contenedor y el contexto próximo. Además, se han posicionado todas las evidencias arqueológicas en el espacio mediante una estación total para su posterior volcado en la planimetría de la cavidad. 


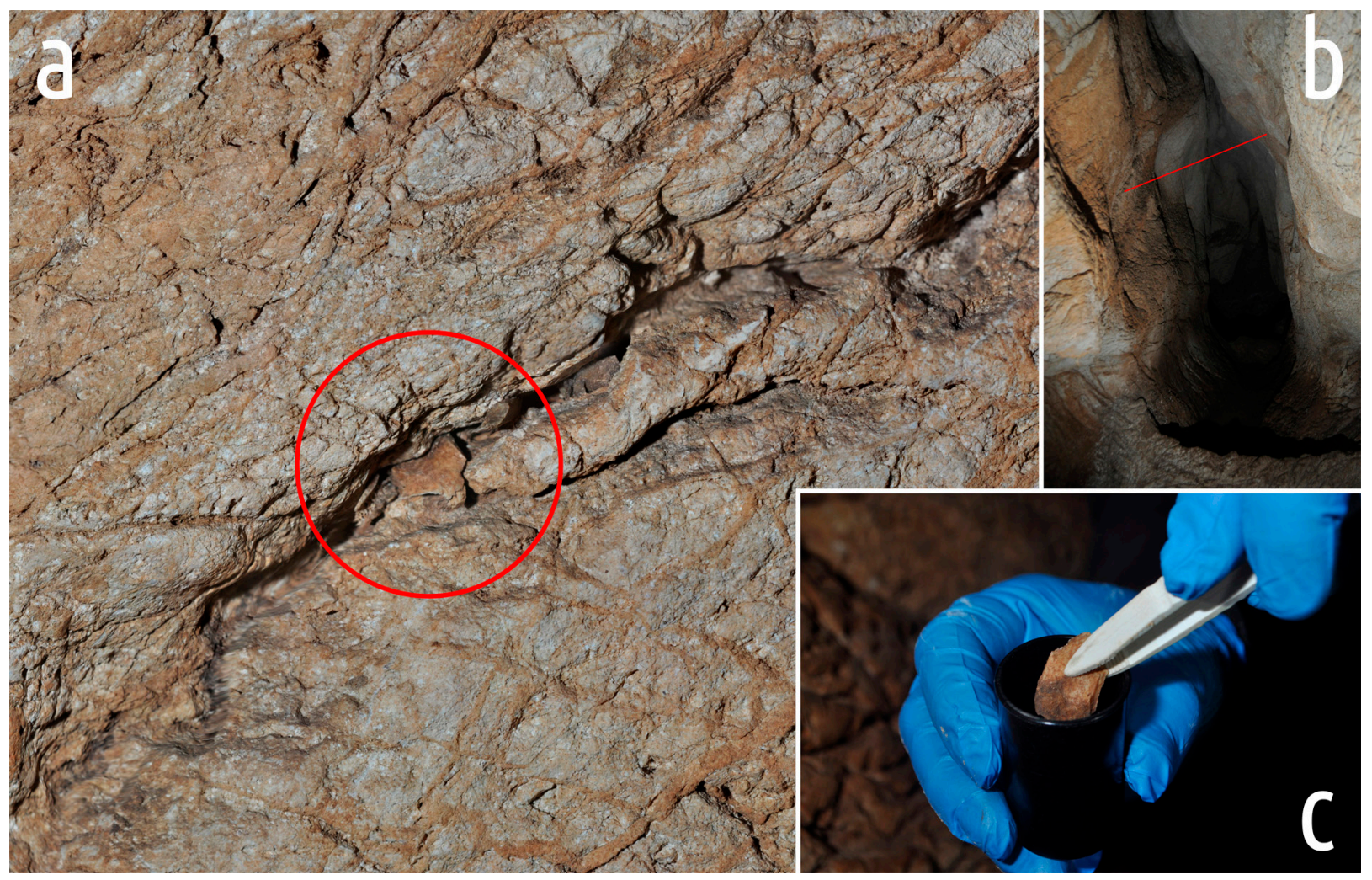

Fig. 3. Proceso de muestreo del hueso insertado PasB.I.04.

Por último, se ha procedido al muestreo (Fig. 3) y tratamiento en el Dpto. de Evolución $\mathrm{Hu}-$ mana del Max Planck Institute for Evolutionary Anthropology-MPI-EVA-, de algunas de las evidencias registradas para la datación por radiocarbono ultrafiltrado, siguiendo el protocolo establecido para tal efecto (Talamo y Richards, 2011). Las muestras de hueso son limpiadas mediante chorro de arena, obteniendo posteriormente $500 \mathrm{mg}$ de polvo de hueso. También son descalcificadas mediante inmersión en $0,5 \mathrm{M} \mathrm{HCl}$ a temperatura ambiente hasta que deja de observarse efervescencia de $\mathrm{CO}_{2}$. El proceso de gelatinización se realiza siguiendo el método Longing con un $\mathrm{PH} 3$ en un calentado de bloque a $75^{\circ} \mathrm{C}$ durante 20 horas. La gelatina es filtrada utilizando un Eeze-Filter ${ }^{\mathrm{TM}}$, -Elkay Laboratory Products Ltd.- para retirar partículas minerales. La gelatina es ultrafiltrada con una membrana Sartorius "Vivaspin 15" de 30 KDalton. Con anterioridad a su uso cada filtro es lavado para eliminar los humectantes contenedores de carbono (Higham et al., 2006). Las muestras son entonces liofilizadas durante 48 horas. Los criterios para la detección de problemas de conservación de colágeno son la ratio $\mathrm{C}: \mathrm{N}, \delta{ }^{13} \mathrm{C}$ y $\delta{ }^{15} \mathrm{~N}$, $\% \mathrm{C}$ y $\% \mathrm{~N}$, y la cantidad resultante de colágeno. Para ser aceptable la muestra debe tener un valor de C:N entre 2.9 y 3,6, y la cantidad resultante de colágeno no debe ser inferior al 1\% (Van Klinken, 1999). Solo tras confirmar que la preservación del colágeno era buena se mandaron las muestras al laboratorio Klaus-Tschira-AMS del Curt-Engelhorn Centre en Mannheim, Alemania, para la medición AMs (Kromer et al., 2013).

\section{Resultados}

Las labores de prospección desarrolladas han permitido localizar un total de 31 objetos depositados en las paredes del Sector в de La Pasiega (Fig. 4). Se distribuyen en 11 paneles desde la entrada 


\begin{tabular}{|c|c|c|c|c|c|c|c|}
\hline Panel & N.o & Овјето & $\begin{array}{c}\text { MEDIDAS } \\
\text { longitud/anchura/suelo }\end{array}$ & Contenedor & Alteración & INSERCIÓN & ORIENTACióN \\
\hline $\mathrm{I}$ & 1 & hueso & $3 \times 0,5 \times 1,85$ & fisura & fractura calcificación & retraído & paralela \\
\hline $\mathrm{I}$ & 2 & hueso & $0,5 \times 2 \times 2,5$ & fisura & & retraído & perpendicular \\
\hline $\mathrm{I}$ & 3 & hueso & $0,5 \times 0,3 \times 1,95$ & fisura & fractura & retraído & perpendicular \\
\hline $\mathrm{I}$ & 4 & hueso & $2 \times 0,5 \times 1,95$ & fisura & & en línea & perpendicular \\
\hline $\mathrm{I}$ & 5 & hueso & $0,5 \times 0,5 \times 1,95$ & fisura & & en línea & perpendicular \\
\hline I & 6 & hueso & $2 \times 1 \times 1,95$ & fisura & fractura & retraído & transversal \\
\hline I & 7 & hueso & $2 \times 1 \times 2,05$ & fisura & & retraído & perpendicular \\
\hline I & 8 & hueso & $1 \times 1 \times 2,05$ & fisura & & en línea & perpendicular \\
\hline II & 1 & hueso & $2 \times 0,5 \times 1,5$ & fisura & fractura & retraído & perpendicular \\
\hline III & 1 & hueso & $2 \times 1 \times 1,7$ & fisura & lengüeta inferior & retraído & perpendicular \\
\hline IV & 1 & hueso & $2,5 \times 0,5 \times 1,8$ & fisura & fractura dientes de sierra & en línea & transversal \\
\hline $\mathrm{V}$ & 1 & hueso & $4 \times 1 \times 1,35$ & fisura & & retraído & paralelo \\
\hline $\mathrm{v}$ & 2 & hueso & $0,5 \times 0,3 \times 1,12$ & fisura & & retraído & paralelo \\
\hline $\mathrm{v}$ & 3 & hueso & $0,6 \times 0,3 \times 1,31$ & fisura & & en línea & perpendicular \\
\hline $\mathrm{v}$ & 4 & hueso & $0,3 \times 0,3 \times 1,32$ & fisura & & en línea & perpendicular \\
\hline VI & 1 & hueso & $2 \times 1 \times 1,38$ & fisura & & en línea & perpendicular \\
\hline VI & 2 & hueso & $1 \times 1 \times 1,39$ & fisura & & en línea & perpendicular \\
\hline VII & 1 & sílex & $-x-x 1,43$ & anfractuosidad & & retraído & - \\
\hline VIII & 1 & hueso & $0,5 \times 1 \times 1,4$ & fisura & & retraído & perpendicular \\
\hline VIII & 2 & hueso & $<1 \times<1 \times 1,4$ & fisura & & retraído & perpendicular \\
\hline VIII & 3 & hueso & $2,5 \times 0,7 \times 1,53$ & fisura & & retraído & paralelo \\
\hline VIII & 4 & sílex & $4 \times 0,7 \times 1,5$ & fisura & $\begin{array}{l}\text { patinado (fragm. proximal } \\
\text { de lámina) }\end{array}$ & retraído & perpendicular \\
\hline VIII & 5 & hueso & $2 \times 0,5 \times 1,49$ & fisura & & retraído & perpendicular \\
\hline IX & 1 & hueso & $2,5 \times 2,5 \times 1,28$ & fisura & & en línea & paralelo \\
\hline IX & 2 & hueso & $3 \times 1 \times 1,62$ & fisura & fractura & aflorante & perpendicular \\
\hline IX & 3 & hueso & $3,5 \times 1 \times 1,12$ & fisura & lengüeta superior & aflorante & perpendicular \\
\hline IX & 4 & hueso & $3 \times 0,4 \times 1,39$ & fisura & & retraído & paralelo \\
\hline $\mathrm{x}$ & 1 & hueso & $2 \times 0,5 \times 1,4$ & fisura & & retraído & perpendicular \\
\hline $\mathrm{x}$ & 2 & hueso & $3 \times 3 \times 1,3$ & fisura & fractura & retraído & perpendicular \\
\hline $\mathrm{XI}$ & 1 & hueso & $1,5 \times 1,5 \times 1,27$ & fisura & & retraído & perpendicular \\
\hline $\mathrm{XI}$ & 2 & hueso & $1 \times 1 \times 1,30$ & fisura & & retraído & perpendicular \\
\hline
\end{tabular}

FIG. 4. Relación de objetos insertados/depositados en las paredes de La Pasiega B.

taponada hasta adentrarse $15 \mathrm{~m}$ en la cavidad. Cuatro de ellos -I, V, VIII y IX- presentan concentraciones de objetos mientras que el resto se encuentran más dispersos. Entre los 31 hay 2 que son piezas de sílex mientras que el resto son fragmentos de hueso. El grado de inserción en la pared, la orientación, las fracturas de los fragmentos y la visibilidad son factores variables que han sido documentados en todos los casos.

Fundamentalmente, nos encontramos ante pequeños fragmentos de hueso menores a los 2 centímetros y, en consecuencia, difícilmente determinables taxonómicamente. Además, debido a su posición expuesta presentan un alto grado de alteración, que se refleja en la pérdida de coloración, de masa y de colágeno. Entre ellos, encontramos algunos ejemplares -9- que presentan fracturas intencionales en lengüeta probablemente producidas en el momento de su inserción, como sucede en otras cavidades -por ejemplo, Isturitz-. Es el único tipo de alteración antrópica detectada, ya que no se ha identificado ningún elemento de industria ósea 
entre los mismos. Además, se han documentado 2 fragmentos de sílex. Uno de ellos, PasB.vir.1, se localiza en el fondo de una oquedad, lo que limita su análisis, en todo caso, parece tratarse de una lasca. Por otro lado, la pieza PasB.viII.4 se ubica mucho más visible en una grieta superficial. Se trata de una lámina cortical desbordante, sobre sílex translúcido probablemente de monte Picota, y presenta huellas de uso macroscópicas en el lado opuesto al dorso cortical (Fig. 5).

Se decidió muestrear, con la autorización correspondiente por parte de la Consejería de Educación, Cultura y Deporte, 4 fragmentos óseos introducidos en las paredes de la cueva -Pasb.r.04, PasB. III.01, PasB.VI.1, PasB.Ix.02-, procediendo a su documentación fotogramétrica previa. Las muestras fueron enviadas al laboratorio Klaus-Tschira-AMS del Curt-Engelhorn Centre en Mannheim, Alemania, para su datación por ultrafiltración. Cada uno de ellos procede de un panel distinto y fueron seleccionados en función de sus propiedades -tamaño, integridad, compacidad, etc.-

Solamente la muestra PasB.I.04 ofreció una cantidad de colágeno suficiente para poder ser datada y arroja un resultado encuadrado en el Paleolítico Superior Final, mientras que las restantes no aportaron resultado alguno al carecer de colágeno suficiente. Ante las escasas posibilidades de éxito decidimos no continuar con el muestreo de más evidencias anteponiendo así su conservación.

El resultado obtenido se corresponde culturalmente con el Magdaleniense Medio (Fig. 6), al que se atribuye también la segunda fase decorativa de la zona de la entrada de La Pasiega B, compuesta fundamentalmente por caballos grabados de pequeño tamaño y muy detallados, que se superponen a las grandes figuras animales rojas de cronología premagdaleniense (González Sainz y Balbín, 2002).
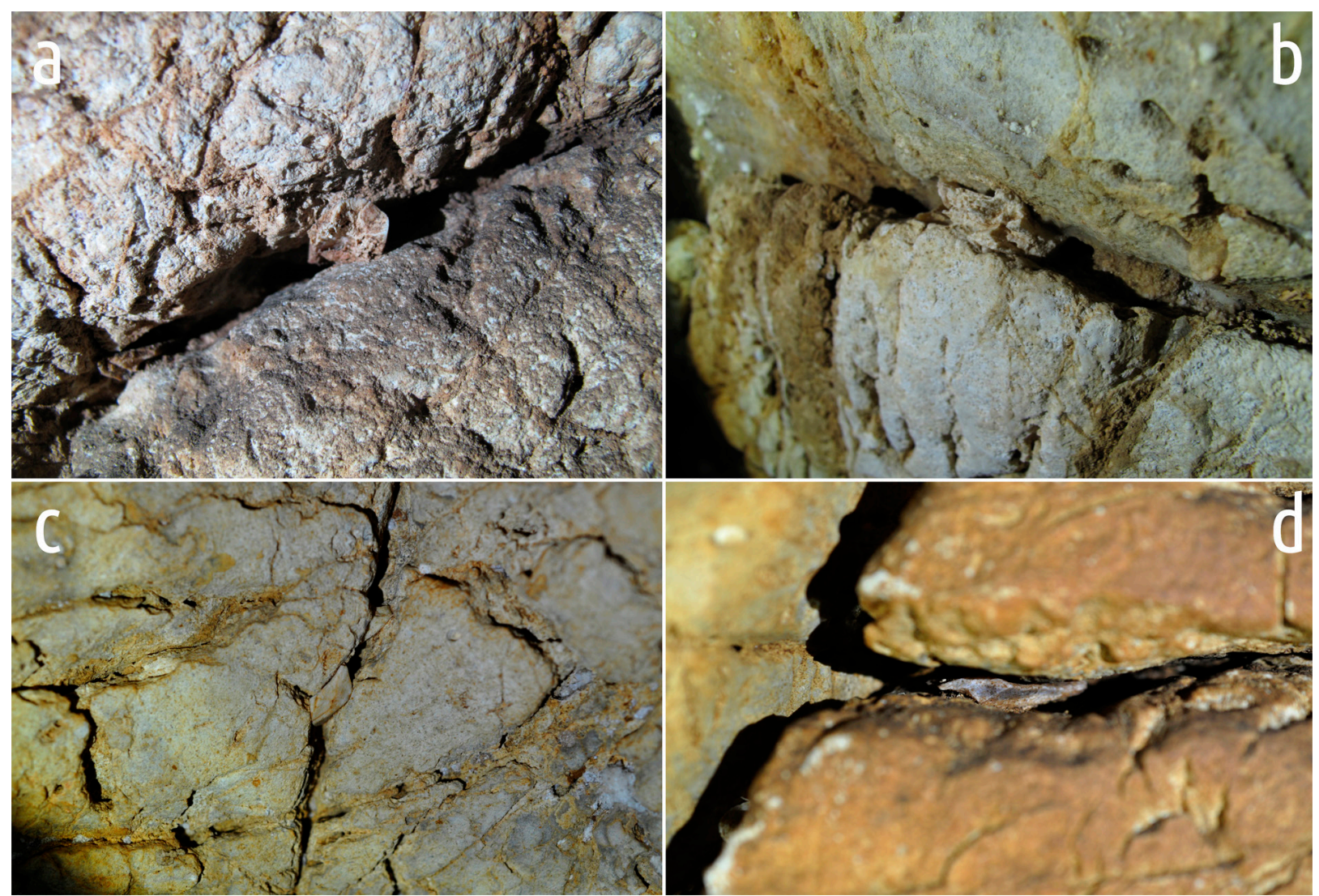

Fig. 5. Huesos: a) Pasb.I.8; b) PasB.IV.1; c) PasB.VI.1-2 y sílex: d) PasB. VII.4, insertados en las grietas de la pared de la cueva. 


\begin{tabular}{|l|c|c|c|c|c|c|c|c|c|c|c|c|c|c|}
\hline MPI Code & $\begin{array}{c}\text { Samples } \\
\text { Code }\end{array}$ & $\begin{array}{c}\text { Used } \\
\mathbf{m g}\end{array}$ & $\begin{array}{c}\text { Yield } \\
\mathbf{m g}\end{array}$ & $\begin{array}{c}\% \\
\text { Yield }\end{array}$ & $\mathbf{\delta}^{13} \mathbf{C}$ & $\mathbf{\delta}^{15} \mathbf{N}$ & $\% \mathbf{C}$ & $\% \mathbf{N}$ & $\mathbf{C : N}$ & $\begin{array}{c}\text { AMS } \\
\text { Lab Code }\end{array}$ & $\begin{array}{c}{ }^{14} \mathbf{C} \\
\text { Age }\end{array}$ & $\begin{array}{c}\mathbf{1 s} \\
\text { Err }\end{array}$ & $\mathbf{6 8 . 2 \%}$ cal BP & $\begin{array}{c}\mathbf{9 5 . 4 \%} \\
\text { Cal BP }\end{array}$ \\
\hline R-EVA1045 & PAS.B.I.04 & 599,5 & 27,7 & 4,6 & $-20,22$ & 3,69 & 42,11 & 15,39 & 3,19 & MAMS-21695 & 14700 & 60 & $18000-17800$ & $18070-17680$ \\
\hline
\end{tabular}

FIG. 6. Fecha de radiocarbono, valores isotópicos, porcentaje de colágeno y relaciones C:N del hueso insertado PasB.I.04. Resultado calibrado con IntCal13 (Reimer et al., 2013) y OxCal 4.3 (Ramsey y Lee, 2013).

\section{Discusión}

En función de lo observado en La Pasiega B, estamos ante el ejemplo más significativo del fenómeno de objetos hincados asociados a arte rupestre de la cornisa cantábrica, y nos obliga además a hacer una reflexión sobre si se trata de un fenómeno más común que ha pasado desapercibido por la falta de interés y de estudios específicos. Hemos enumerado al menos una decena de casos de los que se tiene constancia, aunque, hasta el presente, no se había realizado ningún estudio de conjunto ni tampoco una documentación precisa. A diferencia de las cuevas pirenaicas, la cantidad de objetos suele ser escasa. En todos los casos cantábricos, el gesto concreto de la deposición de objetos aparece espacialmente asociado a los sectores decorados de las cavidades y, por el momento, se desconocen en cuevas sin arte parietal.

Resulta más difícil establecer una cronología ya que los conjuntos artísticos corresponden a periodos muy diversos. Además del ejemplo de La Pasiega в que presentamos en este trabajo, la datación directa se reduce a los casos de Askondo, que sitúa la acción en $23760 \pm 110$ вP y de la cueva de Gargas en los Pirineos, con un hueso asociado a manos positivas datado en $26860 \pm 460$ в (Clottes et al., 1992). El resto de la información disponible es de carácter contextual. En lo que respecta a la cornisa cantábrica, en la cueva de Altxerri в el contexto en el que se localizó la vértebra hincada se restringe cronológicamente al Auriñaciense (González Sainz et al., 2013). En Astigarraga los restos hincados aparecen asociados a trazos pareados, a los que generalmente se les atribuye una cronología solutrense (García Díez, 2011). En Ekain la asociación más probable parece al Magdaleniense, ya que este es el único momento en el que se decora la cavidad (Altuna y Apellaniz, 1978), mientras que en El
Covarón las áreas profundas de la cueva, donde se encuentran los huesos hincados, presentan también un contexto artístico de ese mismo periodo (Arias y Pérez Suarez, 1993). Más complicada resulta la atribución en los casos de la cueva de La Garma por la presencia de paneles decorados en distintas fases del Paleolítico Superior, si bien es cierto que las estructuras de la Zona Iv donde se ubica el sílex hincado parecen tener una cronología sólidamente atribuida al Magdaleniense Medio (Arias, 2009). La cueva de Llonín presenta una amplia y compleja secuencia de ocupación humana que tiene también su reflejo en un panel principal compuesto por sucesivas fases de decoración (Fortea et al., 2004). En ese contexto resulta aventurado atribuir los depósitos de objetos a un periodo concreto sin más argumentos. Por lo tanto, tal y como se reconoce en los Pirineos, Dordoña u otras regiones con elementos similares (Clottes, 1997), se trata de un comportamiento recurrente que pudo prolongarse durante todo el Paleolítico Superior, en paralelo a la propia actividad gráfica.

En el caso que tratamos de La Pasiega в, el resultado radiocarbónico obtenido $-14700 \pm 60 \mathrm{BP}-$ nos permite situarlo en el Magdaleniense Medio, en consonancia con la última fase decorativa diferenciada en ese sector de la cavidad. Debido a la escasez de datos cronológicos obtenida en este proyecto, en gran medida por la mala conservación y el pequeño tamaño de los restos óseos, resulta complicado abordar si se trató de una actividad sincrónica o si la inserción de huesos en las paredes fue una actividad que se prolongó en el tiempo o incluso si se realizó en momentos muy diferentes. Lo cierto es que en ningún otro caso se ha interpretado como una acción diacrónica que pudiera prolongarse durante buena parte del Paleolítico Superior en un mismo yacimiento, como sí sucede con el arte parietal en el caso que tratamos. De cualquier manera, hay 
elementos que permiten proponer, con las limitaciones anteriormente expuestas, la naturaleza sincrónica de esta actividad, estos elementos son el similar estado de conservación de los huesos y la concentración en un espacio bien delimitado en el Sector B de la cueva.

La interpretación de este tipo de evidencias no resulta en absoluto sencilla. La atribución ritual a partir de comparaciones etnográficas (Clottes, 2007; Lewis-Williams, 2002) es la explicación más extendida pero no la única. El estudio sistemático de los casos conocidos en las cuevas de Francia ${ }^{3}$ muestra cierta variabilidad en lo que se refiere a su frecuencia y a su distribución en el interior de las cavidades. De esta manera, podemos diferenciar entre depósitos funcionales y simbólicos. Un primer comportamiento, de carácter colectivo, viene dado esencialmente por esquirlas óseas que se suceden en las fisuras de la pared y que generalmente se concentran en áreas de hábitat con actividades específicas -sería el caso de las cuevas de Isturitz, Enlène, Blanchard, etc.-, por lo que se consideran evidencias funcionales. Incluso se ha propuesto una posible utilidad como sistema de sujeción (Bahn, 2011). Otro tipo de inserciones parecen ser más "confidenciales" puesto que se ubican aisladas en el recorrido subterráneo, protagonizadas, por lo tanto, por aquellas personas que penetrarían hasta los sectores más profundos de las cavidades. Para las inserciones de restos óseos se plantea su relación con ritos de paso -Les Trois-Frères, Le Tuc d'Audoubert, Le Portel, etc.- mientras que, en algunos casos, determinadas inserciones de sílex podrían tratarse simplemente de marcadores y pérdidas relacionadas con la propia progresión espeleológica en el interior de la cavidad -Etxeberri-ko-karbia, Bernifal, Le Tuc d'Audoubert, etc.-. En este sentido, se ha planteado la utilización de herramientas de dicha materia para facilitar la progresión espeleológica en cavidades paleolíticas decoradas (Rios-Garaizar et al., 2015). En otras ocasiones existe una relación espacial directa con la actividad artística al localizarse las inserciones de objetos en los paneles decorados, aunque la vinculación entre ambas actividades

3 Peyroux, op. cit. n. 1. es más difícil de demostrar. En el divertículo axial de Lascaux, en la Galería Jeannel de Le Portel o en varios casos de las cuevas de Les Trois Frères y de Le Tuc d'Audoubert se observan ejemplos de esa relación espacial (Arias, 2009).

En el caso que nos ocupa, la presencia de objetos hincados se concentra junto a los paneles decorados en el Magdaleniense -grabados finos de caballos de pequeño tamaño- y en una fase anterior indeterminada -grandes animales pintados en rojo-. Está también cerca de la entrada a La Pasiega в, espacio este que debió funcionar como lugar de hábitat a tenor de las evidencias arqueológicas excavadas por Carballo y González Echegaray (González Echegaray y Ripoll, 1953/54). La información relativa a la excavación arqueológica desarrollada es muy escasa e imprecisa, pero se evidencian varias fases de ocupación humana. La asociación de este fenómeno a la actividad decorativa dentro de la cueva parece fuera de toda duda habida cuenta la estrecha vinculación de objetos hincados y espacios decorados. La cercanía de esta zona decorada al área de hábitat sugiere que esta zona era accesible a la colectividad del grupo y que todo él pudo intervenir y participar en la actividad artística, en el hincado y depósito de huesos y fragmentos de sílex. Es decir, en el contexto simbólico en el que estas actividades tuvieron lugar. Ese mismo patrón está presente en cuevas como Isturitz y Gargas, donde arte parietal, objetos hincados y hábitat comparten la misma área cercana a la entrada, mientras que en Enlène y La Garma sucede en espacios profundos. Sin embargo, a falta de una investigación más profunda sobre el fenómeno descrito, con los datos disponibles resulta muy complicado ir más allá en la interpretación de esta actividad tan particular.

\section{Conclusiones}

La prospección y documentación de objetos hincados en la cueva de La Pasiega в nos ofrece una importante información de base para el mejor conocimiento de dicho fenómeno en el ámbito cantábrico, donde se trata del primer estudio específico para este tipo de evidencias arqueológicas. 
Además, la posibilidad de situar cronológicamente estas evidencias es un aspecto extraordinario de sumo interés -solamente aplicado con anterioridad en el caso de la cueva de Askondo-, y, aunque la limitación a una sola muestra datable resta posibilidad de contraste y de verificación, la técnica de ultrafiltrado ofrece una precisión máxima.

No cabe duda de que la inserción de objetos en las paredes es un comportamiento del Paleolítico Superior todavía muy mal conocido. La documentación exhaustiva del registro se antoja como un punto de partida para poder afrontar el estudio de este fenómeno tan peculiar de la manera más objetiva y precisa posible.

\section{Bibliografía}

Altuna, J. (1996): "Hallazgo de dos nuevos bisontes en la cueva de Altxerri (Aia, País Vasco)". Munibe (Antropologia-Arkeologia), 48, pp. 7-12.

Altuna, J. (1997): Ekain y Altxerri. San Sebastián: Haranburu.

Altuna, J. y Apellániz, J. M. (1978): "Las figuras rupestres paleolíticas de la cueva de Ekain (Deva)", Munibe, 28, pp. 1-151.

Arias, P. (2009): "Rites in the dark? An evaluation of the current evidence for ritual areas at Magdalenian cave sites", World Archaeology, 41 (2), pp. 262-294.

Arias, P. y Pérez Suárez, C. (1993): "Las pinturas rupestres paleolíticas de El Covarón (Parres, Llanes, Asturias)", Zephyrus, 46, pp. 37-75.

Bahn, P. (2011): "Religion and ritual in the Upper Paleolithic". En Insoll, T. (ed.): The Oxford Handbook of the Archaeology of Ritual and Religion. Oxford, pp. 344-357.

Balbín, R. y González Sainz, C. (1993): "Nuevas investigaciones en la cueva de La Pasiega (Puente Viesgo, Cantabria)", Boletín del Seminario de Estudios de Arte y Arqueología, 59, pp. 9-34.

Balbín, R. y González Sainz, C. (1994): "Un nuevo conjunto de representaciones en el sector D. 2 de la cueva de La Pasiega (Puente Viesgo, Cantabria)", Museo y Centro de Investigación de Altamira, 17, pp. 269-280.

Balbín, R. y GonzÁlez Sainz, C. (1995): "L'ensemble rupestre paléolithique de 'La Rotonda', dans la galerie в de la grotte de La Pasiega (Puente Viesgo, Cantabria)", L'Anthropologie, 99 (2/3), pp. 296-324.
Balbín, R. y González Sainz, C. (1996): "Las pinturas y grabados paleolíticos del corredor B.7 de la cueva de La Pasiega (Cantabria)". En Moure, A. (coord.): El hombre fósil 80 años después. Santander: Univ. de Cantabria, pp. 271-294.

Bégouën, R. y Clottes, J. (1995): "Os plantés et peintures rupestres dans la caverne d'Enlène". En Delporte, H. y Clottes, J. (eds.): Pyrénées préhistoriques. Arts et sociétés. Actes du $118^{e}$ Congrès National des Sociétés Savantes (Pau, 1993). Paris: Cтнs, pp. 283-306.

Breuil, H.; Obermaier, H. y Alcalde del Río, H. (1913): La Pasiega à Puente Viesgo (Santander) (Espagne). Monaco: Chêne.

Bronk Ramsey, C. y Lee, S. (2013): "Recent and planned developments of the program OxCal", Radiocarbon, 55, pp. 720-730.

Carballo, J. (1952): “¿Son auriñacienses las pinturas de la cueva 'La Pasiega'?", Zephyrus, 3, pp. 75-79.

Castillon, R. (1980): "Un autre oiseau à La Pasiega", Travaux de l'Institut d'Art Préhistorique, 22, pp. 157-163.

CLottes, J. (2007): "Un geste paléolithique dans les grottes ornées: os et sílex plantés". En Desbrosse, R. y ThÉvenin, A. (dirs.): Arts et cultures de la préhistoire. Hommages à Henri Delporte. Paris: CTHs, pp. 41-54.

Clottes, J.; Valladas, H.; Cachier, H. y Arnold, M. (1992): "Des dates pour Niaux et Gargas", Bulletin Société Préhistorique Française, 89 (9), pp. 270-274.

Fortea, J.; Rasilla, M. y Rodríguez Otero, V. (2004): "L'art pariétal et la séquence archéologique paléolithique de la grotte de Llonín (Peñamellera Alta, Asturias, Espagne)", Préhistoire, Art et Sociétés, revue éditée par la Société Préhistorique Ariège-Pyrénées, 59, pp. 7-29.

Garate, D. (2010): Las ciervas punteadas en las cuevas del Paleolitico. Una expresión pictórica propia de la cornisa cantábrica. Munibe (Antropologia - Arkeologia), supl. 33. Donostia-San Sebastián.

Garate, D.; Labarge, A.; Rivero, O.; Normand, C. y Darricau, J. (2013): "The cave of Isturitz (West Pyrenees, France): one century of research in Paleolithic parietal art", Arts, 2 (4), pp. 253-272.

Garate, D. y Rios-Garaizar, J. (eds.) (2012): La cueva de Askondo (Mañaria, Bizkaia). Arte parietal y ocupación humana durante la Prehistoria. Kobie (Excavaciones Arqueológicas en Bizkaia), 2. Bilbao: Diput. Foral de Bizkaia.

Garate, D.; Rios-Garaizar, J. y Ruiz, A. (2013): "El arte parietal paleolítico de la cueva de Lumentxa (Lekeitio, Bizkaia)", Kobie (Serie Paleoantropología), 32, pp. 5-28. 
García Díez, M.; Mujika, J.; Sasieta, M.; ArruabarrenA, J. y Alberdi, J. (2011): "La grotte d'Astigarraga (Deba, Guipuzcoa, Spain)”, International Newsletter on Rock Art, 60, pp. 13-16.

González Echegaray, J. y Ripoll, E. (1953/54): “Hallazgos en la cueva de La Pasiega (Puente Viesgo, Santander)", Ampurias, 25-26, pp. 43-65.

González Echegaray, J. y Moure, A. (1971): “Representaciones rupestres inéditas en la cueva de La Pasiega (Puente Viesgo, Santander)", Trabajos de Prehistoria, 28, pp. 401-405.

González García, R. (1987): “Organisation, distribution and typology of the cave art of Monte del Castillo, Spain”, Rock Art Research, 4, pp. 127-136.

González García, R. (1990): "Notas sobre la organización y ubicación del arte paleolítico en las cuevas del monte del Castillo (Puente Viesgo)", Zephyrus, 43, pp. 53-60.

GonzÁlez García, R. (2001): Art et space dans les grottes paléolithiques cantabriques. Grenoble: Jérôme Millon.

GonzÁlez SAinz, C. (2000): "El Megaceros giganteus en la Región Cantábrica. Las representaciones parietales de las cuevas de La Pasiega y de La Garma”, Sautuola, 6, pp. 185-195.

González Sainz, C. y Balbín Behrmann, R. (2000): "Revisión de las representaciones rupestres paleolíticas de la cueva de La Pasiega en el conjunto del monte Castillo. Topografía y documentación artística”. En Ontañón, R. (coord.): Actuaciones Arqueológicas en Cantabria, 1984-1999. Santander: Gob. de Cantabria, pp. 69-73.

González Sainz, C. y Balbín Behrmann, R. (2002): "La Pasiega, A.C.D.P.s.". En Las cuevas con arte paleolitico en Cantabria. ACDPs-Cantabria en imagen. Santander, pp. 165-178.

González Sainz, C.; Ruiz Redondo, A.; Garate, D. e IrIARTe, E. (2013): "Not only Chauvet: dating Aurignacian rock art in Altxerri в Cave (northern Spain)", Journal of Human Evolution, 65 (4), pp. 457-464.

Gorrotxategi, X. (2000): Arte paleolitico parietal de Bizkaia. Kobie, anejo n. ${ }^{\circ}$ 2. Bilbao: Diput. Foral de Bizkaia.

Groenen, M. (2000): Sombra y luz en el arte paleolitico. Barcelona: Ariel.

Heras, C. (1994): "Estudio de la estructuración del espacio artístico en el Arte Paleolítico. La galería 'A' de la cueva La Pasiega”, Museo y Centro de Investigación de Altamira, 17, pp. 281-300.

Higham, T. F. G.; Jacobi, R. M. y Ramsey, C. B. (2006): "AMS radiocarbon dating of ancient bone using ultrafiltration”, Radiocarbon, 48, pp. 179-195.
Hoffmann, D. L.; Pike, A. W. G.; García-Díez, M.; Pettitt, P. B. y Zilhâo, J. (2016): "Methods for U-series dating of $\mathrm{CaCO}_{3}$ crusts associated with $\mathrm{Pa}$ laeolithic cave art and application to Iberian sites", Quaternary Geochronology, 36, pp. 104-119.

Hoffmann, D. L.; Standish, C. D.; García-Díez, M.; Pettitt, P. B.; Milton, J. A.; Zilhão, J.; Alcolea, J. J.; Cantalejo, P.; Collado, H.; De Balbín, R.; Lorblanchet, M.; Ramoz, J.; Weniger, G.-Ch. y Pike, A. W. G. (2018): "U-Th dating of carbonate crusts reveal Neandertal origin of Iberian cave art", Science, 359, 6378, pp. 912-915.

Kromer, B.; Lindauer, S.; Synal, H.-A. y Wacker, L. (2013): "MAMs- A new AMs facility at the Curt-Engelhorn-Centre for Achaeometry, Mannheim, Germany", Nuclear instruments and methods in physics research Section B: beam interactions with materials and atoms, 294, pp. 11-13.

LeWIs-Willians, D. (2002): L'Esprit dans la grotte. La conscience et les origines de l'art. Paris: Édit. du Rocher.

Ochoa, B.; Garrido-Pimentel, D. y García-Díez, M. (2017): "Looking through past records: The use of historical documents in cave art spatial studies and its application to La Pasiega (Puente Viesgo, Cantabria, Spain)”, Quaternary International, 430 (A), pp. 30-140.

Peyroux, M. (2010/11): “Les dépôts d'objets en paroi dans les grottes de Gargas (Hautes-Pyrénées, France): étude et contextualisation du geste”. En Clottes, J. (dir.): L'art pléistocène dans le monde/Pleistocene art of the world/Arte pleistoceno en el mundo. Actes du Congrès IFRAO (Tarascon-sur-Ariège, 2010. Symposium Art pléistocène en Europe). Préhistoire, Art et Sociétés, 6566, pp. 249-271.

Pike, A. W. G.; Hoffmann, D. L.; García-Díez, M.; Pettit, P. B.; Alcolea, J.; De Balbín, R.; González Sáinz, C.; De las Heras, C.; Lasheras, J.-A.; Montes, R. y Zilhâo, J. (2012): "U-series dating of Paleolithic art in 11 caves in Spain”, Science, 336, pp. 1409-1413.

Reimer, P. J.; Bard, E.; Bayliss, A.; Beck, J. W.; Blackwell, P. G.; Bronk Ramsey, C.; Grootes, P. M.; Guilderson, T. P.; Haflidason, H.; Hajdas, I.; Hatté, C.; Heaton, T. J.; Hoffmann, D. L.; Hogg, A. G.; Hughen, K. A.; Kaiser, K. F.; KroMer, B.; Manning, S. W.; Niu, M.; Reimer, R.W.; Richards, D. A.; Scott, E. M.; Southon, J. R.; Staff, R. A.; Turney, C. S. M. y Van der Plicht, J. (2013): "IntCal13 and Marine13 radiocarbon age calibration curves 0-50,000 years cal BР", Radiocarbon, 55, pp. 1869-1887. 
Rios-Garaizar, J.; Perales, U.; Bourrillon, R.; Jiménez-Mena, J.; Garate, D. y Medina-AlCaide, M. A. (2015): "Parietal artistic expression and discreet archeological data inside the paleolithic caves: usewear analysis from Nerja and Etxeberri lithic assemblages", Arkeos, 37, pp. 489-494.

Ripoll, E. (1956): "Nota acerca de algunas nuevas figuras rupestres de las cuevas de El Castillo y La Pasiega (Puente Viesgo, Santander)". En Actas IV Congreso
Internacional de Ciencias Prehistóricas y Protohistóricas. Zaragoza, pp. 301-310.

TAlamo, S. y Richards, M. (2011): “A comparison of bone pretreatment methods for AMs dating of samples >30,000 вг", Radiocarbon, 53, pp. 443-449.

Van Klinken, G. J. (1999): "Bone collagen quality indicators for palaeodietary and radiocarbon measurements", Journal of Archaeological Science, 26, pp. 687-695. 
